\title{
Factores desencadenantes, estructura y función del síntoma fóbico en la infancia: análisis de dos casos clínicos paradigmáticos*1
}

\author{
Julieta De Battista*2 \\ Jesica Varela*3 \\ Mariana Dinamarca*4
}

El objetivo del artículo es analizar dos casos de presentaciones fóbicas en la infancia: Juanito (Freud, 1909) y Sandy (Shnurmann, 1949) de acuerdo a un método clínico. Pudo establecerse que los factores desencadenantes diferían. En el primero se acentúa la carencia paterna y en el segundo la carencia materna. La estructura del síntoma difiere también: es metafórica en el primer caso y metonímica en el segundo. La función del síntoma concierne en ambos casos a la suplencia de la función carente.

Palabras-clave: Psicopatología, infancia, fobia, Lacan

${ }^{*}$ Proyecto de investigación acreditado por UNLP en el Programa de Incentivos a la investigación "Las fobias: ¿síntoma y/o estructura? Función de suplencia y nominación” dirigido por Graziela Napolitano.

Financiamiento de la UNLP, el CONICET y el Ministerio de Salud de la Provincia de Buenos Aires.

*2 Universidad Nacional de La Plata (La Plata, Argentina); CONICET (Buenos Aires, Argentina)

* 3,4 Universidad Nacional de La Plata (La Plata, Argentina). 


\section{Introducción}

Este trabajo propone interrogar las diferencias en la estructura y función del síntoma fóbico en dos casos de niños: Juanito - caso analizado por Freud en 1906 - y Sandy — caso presentado por una discípula de Anna Freud, Anneliese Shnurmann en 1946 - , a partir de un análisis de los factores desencadenantes de las crisis.

El análisis del caso Juanito condujo a Freud a postular que la fobia infantil era una formación sustitutiva que cumplía la función de tramitar los componentes del Complejo de Edipo (Freud, 1926/1998), hipótesis que luego extendió por ejemplo al caso del Hombre de los Lobos y que se generalizó entre sus discípulos en la explicación de la fobia. Siguiendo los pasos de Freud, Lacan le otorgó al síntoma fóbico de Juanito una estructura metafórica. En cambio con respecto a la función, sostuvo que se trataba de suplir al significante del padre simbólico en un contexto de carencia de intervención del padre real ante los intentos de seducción del niño con respecto a la madre, reanimados por la presencia efectiva de las primeras erecciones (Lacan, 1956-57/1994).

De esta manera, la explicación del síntoma fóbico queda ligada en ambos autores a los avatares de la función del padre. Si bien la presentación del síntoma en Sandy puede parecer semejante a la de Juanito (ambos son niños, el malestar se presenta bajo la forma del miedo al caballo en Juanito y del miedo al perro en Sandy), en el segundo caso no es la carencia paterna lo que operaría como factor desencadenante de la fobia, sino más bien la carencia de la madre y la 


\section{ARTIGOS}

privación de pene, por tratarse de una niña. Este caso permite así interrogar lo establecido con respecto al caso Juanito, que se ha utilizado como modelo para entender el surgimiento de fobias en la infancia (Miller, 1995; Napolitano \& Acuña, 2003).

Esta investigación contribuye a complejizar el conocimiento sobre las distintas estructuras y funciones que podría tener en la economía psíquica la presentación del malestar bajo la forma de miedos y fobias en los niños e intenta producir un aporte a la lectura clínica de este tipo de material que pueda impactar en la dirección de la cura.

Metodológicamente la investigación consistió en realizar una comparación de ambos casos clínicos para establecer similitudes y diferencias. Luego se propone otra lectura del material clínico a partir de los siguientes operadores teóricos definidos por Lacan en 1955-1958: castración, privación, frustración, función de la madre, función del padre, posición del niño con respecto a la pareja parental, relación al falo como referencia simbólico-imaginaria. El uso del material clínico no se reduce a la ilustración de conceptos sino que incluye una dimensión heurística en la elaboración de los mismos.

El marco teórico elegido en esta investigación es la relectura que Lacan hace de Freud en los años 1950. Más específicamente, en 1956-1957 Lacan dio su seminario sobre "La relación de objeto y las estructuras freudianas", en el curso del cual comentó los dos casos que nos ocupan en este artículo (Lacan, 1956-57/1994). Este seminario precede al de "Las formaciones del inconsciente" donde Lacan desplegó su fórmula de la metáfora paterna y construyó el grafo del deseo (Lacan, 1957-58/1998). Un eje común atraviesa estos seminarios: el cambio de axiomática de las leyes de la palabra a las leyes del lenguaje (metáfora/metonimia) y el valor que adquiere la función significante del padre. Es sobre todo este aspecto el que se ha retenido y jerarquizado en las elaboraciones de la fobia que se realizaron posteriormente (Miller, 1995; Napolitano \& Acuña, 2003).

No obstante, es posible demostrar que la inclusión del caso Sandy permitiría pensar una función de la fobia que no está ligada directamente a la función del padre, sino a la de la madre en la instauración de los inicios de un orden simbólico.

Las preguntas que orientaron la investigación podrían formularse de la siguiente manera: ¿qué función cumple la aparición del síntoma fóbico en la infancia? Su estructura ¿es siempre metafórica? ¿hay diferencias en los factores desencadenantes, en la estructura y función del síntoma fóbico según se presente en un niño o en una niña? 


\section{Marco teórico}

En ese momento de su obra, Lacan señaló que el psicoanálisis de postguerra se había desviado en su modo de entender lo que se dio en llamar "relación objetal" reduciéndola a la relación del sujeto con su entorno (Lacan, 1956-57/1994). Ya desde sus formulaciones sobre el estadio del espejo Lacan complejizó la relación del sujeto con el otro, al distinguir primero yo de sujeto y luego el otro imaginario del Otro simbólico (Lacan, 1954-55/1978). El movimiento siguiente fue incluir al falo como elemento tercero en la composición del Edipo, al otorgarle una estructura cuaternaria: niño, madre, falo, padre.

La falta de objeto es el resorte fundamental de la relación del sujeto con su mundo. Ahora bien, esta falta de objeto presenta diferentes niveles ordenados de acuerdo a los registros que intervienen. Lacan conceptualizó a la castración en el plano de una deuda simbólica, una ley primordial, cuyo objeto es imaginario. La frustración, en cambio, respondería a una lesión o daño imaginario de un objeto que es real y se presenta por lo tanto no como una ley primordial, sino como una exigencia sin ley. Por último, la privación aparece como una falta real de un objeto simbólico que Lacan atribuye a un agujero real, cuyo ejemplo paradigmático es la condición de la mujer.

De esta manera, la falta de objeto es el elemento organizador de la experiencia que ya no es entendida en términos imaginarios sino desde una primacía del significante. La estructura del mundo humano es la de la falta de objeto. En este contexto el niño se le presenta a Lacan como el sujeto elegido de la dialéctica de la frustración, concepto que por otra parte había polarizado las lecturas post-freudianas y que Lacan pretende rectificar para poder hacerlo utilizable (Lacan, 1956-57/1994). Sin embargo, las lecturas posteriores de estos conceptos privilegiaron la vertiente de la castración, sin retomar la cuestión de la frustración y la privación.

Las preguntas que orientaron a Lacan en estas formulaciones pretenden dirimir cómo es que el niño se introduce en un mundo simbólico que está operando antes de su llegada, cómo el niño es llevado a simbolizar lo real, cómo la frustración introduce el orden simbólico. La hipótesis fuerte de Lacan, que ha sido poco retomada, es que el niño es introducido en la discordancia radical por la cual para la madre no es sólo niño sino falo después de una época de "simbolización primera" (Lacan, 1956-57/1994, p. 57) que en cierto sentido está más allá y más acá de la función del padre. Aquí es donde la fobia y el fetiche aparecen como dos soluciones diversas al problema de las relaciones de la madre con el niño. La relación con el falo no se daría de forma espontánea y directa. 


\section{ARTIGOS}

\section{Análisis de casos clínicos}

\section{El caso Juanito}

Cerca de cumplir cinco años Juanito comienza a padecer un síntoma fóbico que lo limita en sus desplazamientos, cuyo contenido es el miedo a ser mordido por un caballo (Freud, 1909/2000). Las condiciones previas a la eclosión de la fobia hablan de un niño feliz, hijo único, criado en un ambiente permisivo, "no está frustrado ni privado de nada" (Lacan, 1956$-57 / 1994)$. Freud señala que en un inicio el niño está muy interesado en su pene, momento normal del desarrollo que luego nombrará como premisa universal del pene (Freud, 1925/1993b; Freud, 1923/1993a). Estas investigaciones infantiles son correlativas de los comienzos de la masturbación y las primeras amenazas, proferidas en este caso por la madre, que adquirirán eficacia retroactivamente.

Lacan subraya otro elemento no tan privilegiado por Freud: el hecho de que el niño comparta el lecho conyugal con la madre, quien desoye las intervenciones del padre al respecto. Jerarquiza entonces que se trata de una madre que no hace caso de la palabra del padre (Lacan, 1958/1966), que se presenta como fálica ante el niño y para quien este padre no parece contar demasiado en su deseo. El padre se presenta imaginariamente como un "buen tipo", un "pobre hombre", amado por su hijo pero que resulta no estar a la altura de la función del padre simbólico, que finalmente cumplirá Freud al apuntalar a este padre real en el ejercicio de una función signada por su carencia (Lacan, 1956-57/1994, p. 223).

Juanito se encuentra entonces en pleno juego de seducción de la madre, que Lacan conceptualiza como un juego del señuelo donde el niño está entrampado en una relación imaginaria mediante la cual asegura a la madre que puede colmarla en cuanto a su deseo, es decir, en cuanto a lo que le falta, siendo el falo (Lacan, 1956-57/1994). Este lugar en el "paraíso del señuelo" (p. 228) comienza a ser conmovido con el nacimiento de su hermana, cuando Juanito tenía tres años y medio. Si bien Freud considera al nacimiento de la niña como un acontecimiento central para la constitución de la fobia, Lacan sostiene que lo que pone término a esta relación ocasionando el desencadenamiento de la fobia es la aparición en la escena del "pene real", es decir las primeras erecciones y la masturbación.

La entrada en juego del goce autoerótico transforma el "paraíso del señuelo" en una trampa: no es lo mismo jugar imaginariamente a ser el falo 
que tener algo real para ofrecer. Esta discordancia es lo que para Lacan produce la emergencia de la angustia que antecede a la conformación del síntoma fóbico. A diferencia de Freud, para Lacan el factor desencadenante de la fobia es la emergencia del pene real - que se presenta al niño como algo totalmente ajeno, invasivo, perturbador, fuera del cuerpo. Esta aparición instaura el drama (Lacan, 1956-57/1994).

Precisamente en este momento la intervención del padre simbólico encarnada en un padre real hubiese permitido producir un efecto normativizante. Y es justamente aquí donde se revela la carencia de la función paterna en el caso Juanito. El síntoma fóbico viene a suplir esta carencia, convirtiendo la angustia en miedo y, al modo de un parapeto, organiza un mundo habitable, protegido de la emergencia subrepticia de la angustia (Freud, 1915/2000b).

Para Lacan, la función de la fobia es la de suplir al padre simbólico, que no opera a falta de la intervención de un padre real que encarne la función en el momento en que Juanito se encuentra entrampado en el juego del señuelo con la madre. La estructura de la fobia opera por sustitución metafórica constituyendo al caballo en el elemento organizador; el significante "para todo uso" (Lacan, 1956-57/1994, p. 289) que sustituye al significante del padre, y gracias al cual, el niño puede simbolizar la castración. De este modo, la fobia tiene una función estructurante, organizadora del mundo del niño, el cual se le aparece puntuado por toda una serie de señales de alarma que lo reestructuran. El significante fóbico se convierte en punto de referencia en el orden simbólico. Este sostén fóbico es momentáneo y se vuelve inútil a medida que el niño va desplegando toda una serie de fantasías.

Esta lectura del caso Juanito enfatiza como factor desencadenante la aparición del pene real en un contexto donde el niño se encontraba en el juego del señuelo con la madre, presentándose como el falo que podría colmarla. En esta situación en la que el niño no estaba ni frustrado, ni privado de nada, el síntoma fóbico cumple la función de suplir al significante del padre simbólico que opera justamente simbolizando la castración. La fobia no hubiera sido necesaria si el padre real hubiera podido efectivizar esa operación encarnando la función.

Por lo tanto, las coordenadas de la eclosión de la fobia en Juanito podrían formularse así: juego del señuelo con la madre (niño falo), aparición del pene real, carencia de intervención del padre real en la encarnadura de la función del padre simbólico. Esta constelación se ha tomado como modelo en la lectura clínica de las fobias en la infancia. No obstante el caso Sandy parece objetarla, o al menos introducir otros aspectos no suficientemente resaltados. 


\section{ARTIGOS}

\section{El caso Sandy}

En el caso de Sandy (Shnurmann, 1949/2002) Lacan habla de una fobia "al límite de la relación edípica", una fobia transitoria e inicial que no llega a cristalizar (Lacan, 1956-57/1994, p. 84). Hay que destacar que el caso fue organizado por Shnurmann en una sucesión cronológica que parece ofrecer una fidelidad ejemplar, pero que está atravesada por la hipótesis de la psicogénesis. Lacan desmenuza el solapamiento producido entre privación, frustración y castración para hacer otra lectura que retomaremos en función de situar las distinciones entre ambos casos.

La configuración de partida de Juanito presenta notables diferencias con el caso Sandy: en principio se trata de una niña, por lo tanto se encuentra privada en lo real de pene. Además de la diferencia de sexos, hay también una diferencia con respecto a la edad en que el síntoma fóbico se presenta: la niña tiene exactamente dos años y cinco meses al momento de inicio de esta "casi" fobia que dura sólo un mes, Juanito tenía casi cinco años cuando la fobia se instala y dura varios meses.

Estas diferencias se traducen también en una distinción en las coordenadas subjetivas de aparición del padecimiento. Lacan acentúa que la presencia de las primeras erecciones tuvo un rol preponderante en la eclosión de la fobia de Juanito, así como la presencia de la madre sin la intervención del padre. En Sandy los factores desencadenantes son otros: la ausencia de la madre, debido a una operación quirúrgica, y la ausencia de pene, aunque no de masturbación (Shnurmann, 1949/2002).

Con respecto a la función de la madre, hay que destacar que Sandy no vivía con ella, sino que estaba bajo tutela en la residencia infantil de Hampstead, creada por Anna Freud para alojar a los niños durante la segunda guerra mundial (Freud, A. 1943). Es allí donde a partir de sus dos años queda al cuidado de una discípula de Anna Freud, Anneliese Shnurmann, quien realiza la observación.

La madre de Sandy trabajaba como jefa de refugio y demostraba cierta predilección por las tareas y el vestuario masculino, de hecho había trabajado como conductora de ambulancias. Esto no le impedía desempeñarse como una "madre consagrada" a los ojos de Shnurmann y Sandy se mostraba muy apegada a ella. Visitaba a la niña por las noches, le llevaba chocolates y se ocupaba de acostarla y de bañarla, cuando la encontraba despierta. Esto era así desde que Sandy era una recién nacida, más precisamente desde sus siete semanas de vida, momento en que es recibida en la Residencia. 
El padre de Sandy había muerto en un accidente en el ejército antes de que ella naciera. Sandy tenía dos hermanos que tampoco vivían con ella ni con la madre: la mayor tenía siete años al nacer Sandy y murió cuando ésta tenía dos. El hermano era dos años mayor que ella y cobrará un papel relevante posteriormente a la resolución de la fobia.

Por lo tanto, la constelación que antecede el inicio de la fobia en Sandy está compuesta por la ausencia de la madre, la muerte de la hermana y la muerte del padre, es decir está signada más bien por las pérdidas que por las presencias. A diferencia de Juanito, donde se resaltan las presencias: la del nacimiento de la hermanita para Freud, la de la madre y el pene real para Lacan. En Juanito queda enfatizada la carencia paterna y en Sandy en cambio es la ausencia de la madre lo que cobra mayor valor en la observación, junto a la ausencia de pene. Tampoco se ha destacado suficientemente a nuestro entender el impacto de la muerte de la hermana en la economía psíquica de la madre y por lo tanto en el lugar que Sandy podía ocupar en su deseo. Entendemos que este comentario tiene su valor, dado que el inicio de la fobia se produce pocos meses después.

Shnurmann privilegia en la observación el papel del descubrimiento 410 de la diferencia de sexos y se aboca a subrayar todo lo que parecería ser indicio del impacto que ese descubrimiento le habría producido: ver a un niño hacer pis, querer hacer pis de pie, inspección de sus genitales, preguntas por las bombachas. Incluso Shnurmann llega a completar el texto de la fobia forzándolo al decir que el temor era a que el perro le arrancara los genitales (cuestión que parece deducir del comportamiento y que resulta muy atravesada por sus supuestos, ya que Sandy recién está comenzando a hablar). Sin embargo, hay otros elementos que vale la pena destacar. Pero reconstruyamos antes en qué punto se encuentra Sandy en el momento en que la fobia aparece.

En la observación se señala que poco antes de los dos años el juego con la madre era de tipo presencia-ausencia, que Sandy ejercitaba con otros ofreciéndoles objetos que luego les quitaba. La madre jugaba con ella a hacerla dudar sobre si iba a verla o no, o a quitarle y darle el chocolate que le llevaba. Shnurmann observa que estos juegos no estaban desprovistos de un componente agresivo en el trato brindado por la madre que se transmudaban en ataques agresivos de la niña hacia los otros niños.

Lacan señala entonces que la función de la madre simbólica está operando y que Sandy se encuentra en esta primera "escansión del llamado" que posibilita el inicio del orden simbólico, por el cual una relación real puede conectarse a una relación simbólica. La pregunta que aquí resulta de interés 


\section{ARTIGOS}

para Lacan es cómo se introduce el niño en la oposición presencia-ausencia, cebo de la instauración del orden simbólico. Dicho de otra manera, cómo se produce el momento de viraje en que la relación primordial con la madre se abre a una relación más compleja, dialéctica (Lacan, 1956-57/1994, p. 70).

En función de estas preguntas la observación del caso Sandy se vuelve esencial ya que es en este contexto de una primera simbolización, de la instalación de la oposición presencia-ausencia que se produce el accidente que cobra relevancia en el desencadenamiento de la fobia: la madre se ausenta a causa de una enfermedad que requiere de una intervención quirúrgica y regresa luego bastante desmejorada, con dificultades para caminar, ya no puede ocuparse de acostar a Sandy, ni de bañarla como antes hacía. Lacan se pregunta: ¿qué ocurre si la madre ya no responde a la llamada del sujeto? (p. 70).

Si eso sucede la madre cae de su posición de objeto ausente-presente para pasar a ser real y convertirse en una potencia: los objetos que hasta entonces eran objetos de satisfacción se convierten a partir de esta no respuesta al llamado en objetos de don y pueden cambiar de estatuto al tomar ellos mismos la connotación de presencia-ausencia ya que dependen de ese objeto real que es ahora la potencia materna. La madre simbólica se ha convertido en real y por este vuelco el objeto real se ha convertido en simbólico: "El objeto vale como testimonio del don proveniente de la potencia materna (...) simboliza una potencia favorable" (p. 71). La madre ausente-presente ha transferido esta característica a los objetos: puede darlos o no darlos. De ahí que las carencias de la omnipotencia materna cobren singular relevancia en el desarrollo del niño.

El caso Sandy parece mostrar muy bien este momento. En pleno juego de presencia- ausencia y del rol de la madre simbólica instalado se produce la ausencia repentina de la madre por la enfermedad: ya no responde al llamado. Al volver a visitar a Sandy, la madre está visiblemente desmejorada: la enfermedad ha atentado contra su potencia, ya no es seguro que ella pueda darlo.

En este punto es interesante resaltar el movimiento de restitución del estado previo que realiza la niña mediante la fobia. Sandy toma nota de la ausencia y repite "Mi mamá está ahí" cuando pasa por la sala en la que se encontraron. Una semana después de esta ausencia de la madre estallará la fobia bajo la forma de una pesadilla de la que decanta el miedo al perro.

Lacan subraya que la visión del genital masculino, el afalicismo, no bastó para producir la fobia, sino que fue necesaria la ruptura en el ritmo alternado de las idas y venidas de la madre y el regreso de la madre afectada en su potencia para que surja la fobia. Es decir, la fobia no se hace necesaria 
ante el descubrimiento de la diferencia de los sexos, cuestión que se corrobora luego en el material. Cuando la madre decide retirar a la niña de la residencia recupera a su otro hijo y se casa. En este contexto de reconstitución familiar Shnurmann observa que las peleas con el hermano rondaban en torno al carácter apeniano de Sandy y eso la lleva a sospechar que el terreno de esa conflictiva debería desencadenar nuevamente la fobia. Pero esto no ocurre.

El devenir del caso nos revela entonces que el surgimiento de la fobia no está tan atado al descubrimiento de la diferencia entre los sexos como al encuentro con la caída de la potencia materna. La fobia sólo se vuelve necesaria en el punto en que la madre aparece como alguien que podría faltar, no volver, y al regresar lo hace debilitada, afectada en su potencia.

Lacan (1956-57/1994, p. 75) precisa entonces que la fobia se vuelve necesaria en cuanto a la madre le falta el falo, en el momento en que Sandy ha perdido ese punto de amarre que había encontrado en esta primera simbolización de la oposición ausencia-presencia.

De esta manera, la cuestión de la privación es reformulada por Lacan dado que no se la puede pensar únicamente en un plano real, el de la ausencia de pene y el consecuente supuesto impacto de la visión del genital masculino, sino que "Una privación sólo puede concebirla efectivamente un ser que articula algo en el plano simbólico" (p. 102). La impotencia de la madre aparece justo en el momento en que Sandy comenzaba a incluirse en la estructura simbólica primitiva de las ausencias y presencias, en la relación con lo que para la madre es el falo. Es justamente en ese momento que se produce en lo real el desfallecimiento fálico de la madre.

Por lo tanto vemos así que el inicio de la fobia de Sandy no presenta las mismas coordenadas que en Juanito. Allí donde en éste encontramos al niño entrampado en el juego de hacerse el falo para la madre, ocupando el lugar de objeto metonímico del deseo de esta madre fálica; en Sandy sin embargo aparecen los efectos de la carencia fálica de la madre. En cierto sentido, lo accidental en Juanito no se ubica en este registro sino en la carencia del padre, suplida metafóricamente por la fobia. Sandy en cambio sólo parece poder llegar al punto de la girl-phallus, la preferida de la madre que despierta incluso la rivalidad del hermano, valiéndose de la fobia.

El caso Sandy parece concluir con la niña jugando a ser el falo de la madre, es decir allí donde Juanito empieza. El rol de la fobia es diferente en ambos casos.

Volviendo a la observación entonces, la angustia aparece luego de la venida de la madre afectada en su potencia y se manifiesta justamente al 


\section{ARTIGOS}

momento de acostarse: Sandy está inquieta y no sabe por qué. Tiene entonces la pesadilla con el perro en la cama, pero esta localización tampoco la tranquiliza, llora una hora entera y no pueden calmarla. Es de notar que los pocos significantes que se entraman en este inicio de fobia conciernen al ir y venir, las oposiciones: el perro viene, el perro está en la cama, lo busca bajo el colchón, está en el otro refugio, "no cama, perrito viene", "no refugio perrito, agua Sandy, no agua perrito", "mi mamá enferma, mi mamá vuelve, mi mamá se va, mi mamá vuelve, mi mamá se marcha”, se reitera el juego de las presencias y ausencias pero ahora con el perro.

El perro parece convertirse en un punto de amarre ante la ausencia de la madre. Incluso cuando Shnurmann alienta la identificación de Sandy con el perro al verla ladrar, la niña se encarga de recuperar la oposición: no quiere hacer de perro, quiere hacer de gato, no pasea a un perro en su cochecito pasea a un "minino". Los momentos de mayor angustia parecen vincularse a la constatación de la ausencia de la madre, dado que se producen en los momentos que antes compartía con ella: acostarse y bañarse. Allí no aparece la madre, pero sí el perro.

El miedo se traslada luego del perro en la cama al perro en la calle, pero esto no parece tener una función de organización del mundo, ni se observa que la fobia funcione al modo del parapeto como en Juanito.

Lacan (1956-57/1994, p. 102) remarca que la fobia desaparece cuando Sandy se reintegra en una familia completa. Simplemente se diluye con el regreso de la madre que la lleva a vivir con ella, para luego casarse y reconstituir la familia junto con el hermano. Shnurmann señala sin embargo que el drama pasó a ser ahora la separación de la madre: duerme con ella, no quiere dejarla para ir la escuela, hace grandes escándalos cuando ella se va, se sabe la preferida de la madre y despierta los celos de su hermano mayor. En este caso, la función del padre simbólico no parece estar obstaculizada por la carencia a nivel del padre real. El nuevo marido de la madre parece estar a la altura de la función y la crisis fóbica no se reitera.

En cierto sentido la fobia ha permitido que Sandy llegara al punto donde la observación de Juanito comienza: no se la puede sacar de la cama de la madre. Pero en Juanito la fobia fue necesaria para que saliera de allí y a la inversa en Sandy se volvió necesaria para que entrara allí. Podríamos pensar aquí en una traducción clínica de la diferencia de los sexos. Lacan destaca que en Sandy la fobia operó un desplazamiento necesario cuyo motor no fue el hecho de que ella no tuviera el falo sino que su madre no podía dárselo. De esta manera el hecho de convivir ahora con un padre y un hermano vuelve 
innecesaria la fobia que surgió según Lacan (1956-57/1994) para "suplir los elementos falóforos ausentes en el circuito simbólico" (p. 102).

\section{Conclusiones}

Del análisis de los casos clínicos propuestos de acuerdo a los operadores teóricos señalados puede deducirse que hay una diferencia en la estructura y función del síntoma fóbico. El reconocimiento de esta diferencia podría resultar útil clínicamente no sólo a nivel del diagnóstico diferencial sino también en la dirección de la cura.

En el caso de Juanito, el surgimiento de la fobia ofrece una solución sintomática a la angustia que emerge ante la siguiente conjugación de factores: niño en posición de falo entrampado en el juego del señuelo con la madre, madre en posición fálica que desoye las palabras del padre, aparición de las primeras erecciones y la masturbación, falla en la intervención del padre simbólico debida a una carencia de encarnadura de la función en el padre real. El síntoma fóbico presenta una estructura metafórica (sustitución del significante del padre simbólico por el caballo) y cumple una función de suplencia. Esta configuración metafórica aporta un plus: el objeto fóbico opera organizando un mundo y le permite a Juanito ubicarse en relación a la sexuación.

En el caso de Sandy las coordenadas de eclosión de la angustia y la posterior fobia difieren: niña en juego de ausencia y presencia con la madre, interrupción abrupta debida a la caída de la potencia materna, ausencia de padre real. La fobia revierte con el regreso de la madre y su casamiento, la niña cambia de posición para ubicarse en el juego del señuelo con la madre. Este síntoma fóbico no permanece y en su transitoriedad presenta una estructura metonímica, opera por desplazamiento y no por sustitución, por lo tanto no aporta un plus de organización del mundo. Cumple una función de punto de amarre en esa primera constitución del mundo simbólico donde la función de la madre es esencial. Opera entonces supliendo la presencia fálica de la madre desfalleciente y conduce a que la niña pueda ubicarse como objeto metonímico del deseo materno, condición que constituye el punto de partida de la observación de Juanito. Esta operación metonímica es la condición previa necesaria a la instauración de la metáfora (Lacan, 1955-56/1981).

Podemos entonces deslindar una estructura de la fobia que no opera por sustitución de significantes sino por desplazamiento (el miedo al perro 


\section{ARTIGOS}

sostiene la alternancia de ausencias-presencias que antes procuraba la madre, pero que se vio interrumpida por su no respuesta al llamado). Se trata más bien de una estructura metonímica y no metafórica cuya consecuencia no aporta un plus en la organización del mundo sino que favorece la asunción del niño como objeto metonímico del deseo materno. Con respecto a la función, no se trataría como en el caso Juanito de la suplencia de la carencia paterna, sino de suplir la carencia en la potencia fálica de la madre.

De este análisis puede deducirse entonces que los síntomas fóbicos en la infancia no presentan siempre una estructura metafórica ni tienen por función suplir al padre simbólico ante una carencia del padre real. También pueden presentarse síntomas fóbicos de estructura metonímica, cuya función es apuntalar el juego de ausencias y presencias que reconoce en la madre simbólica a su agente esencial. En este caso el factor desencadenante de la fobia no sería la carencia paterna sino la materna. Clínicamente la diferencia de estructura y función halla expresión en el potencial organizador del mundo que introduce el síntoma de estructura metafórica y en la condición de posibilidad que ofrece el síntoma metonímico para que el sujeto pueda ubicarse con respecto al deseo materno.

Queda sujeto a discusión si en el segundo caso convendría conservar el término de "síntoma" o sería conveniente proponer el de "miedo" para referirse a estas presentaciones del malestar, subrayando de esta manera la distinción entre una estructura metafórica y sus potenciales de organización y una estructura metonímica que opera como condición de posibilidad de instauración de la metáfora.

\section{Referencias}

Freud, A.; Burlingham, D. (1943). War and children. London: Allen and Unwin.

Freud, S. (2000a). Análisis de la fobia de un niño de cinco años. In Obras completas (t. X, pp. 1-117). Buenos Aires: Amorrortu. (Trabalho original publicado en 1909).

Freud, S. (2000b). Lo inconsciente. In Obras completas (t. XIV, pp. 153-214). Buenos Aires: Amorrortu. (Trabalho original publicado en 1915).

Freud, S. (1993a). La organización genital infantil (una interpolación en la teoría de la sexualidad). In Obras completas (t. XIX, pp. 141-150). Buenos Aires: Amorrortu. (Trabalho original publicado en 1923). 


\section{ARTIGOS}

Freud, S. (1993b). Algunas consecuencias psíquicas de la diferencia anatómica entre los sexos. In Obras completas (t. XIX, pp. 259-276). Buenos Aires: Amorrortu. (Trabalho original publicado en 1925).

Freud, S. (1998). Inhibición, síntoma y angustia. In Obras completas (pp. 71-163). Buenos Aires: Amorrortu. (Trabalho original publicado en 1926).

Lacan, J. (1978). Le séminaire. Livre 2. Le moi dans la théorie de Freud et dans la technique de la psychanalyse. Paris: Seuil. (Trabalho original publicado en 1954-55).

Lacan, J. (1981). Le séminaire. Livre 3. Les psychoses. Paris: Seuil. (Trabalho original publicado en 1955-56).

Lacan, J. (1994). Le séminaire. Livre 4. La relation d'objet. Paris: Seuil. (Trabalho original publicado en 1956-57).

Lacan, J. (1998). Le séminaire. Livre 5. Les formations de l'inconscient. Paris: Seuil. (Trabalho original publicado en 1957-58).

Lacan, J. (1966). D’une question préliminaire á tout traitement possible de la psychose. In Écrits (pp. 531-583). Paris: Seuil. (Original publicado en 1958).

Miller, J.A. (1995). Le cas Sandy selon Jacques Lacan. Bulletin Groupe Petite enfance, Paris, 6/7: 5-19.

Napolitano, G.; Acuña, E. (2003). El caso Sandy... una fobia en tiempos de guer-

ra. XIII Encuentro Internacional del campo freudiano. Disponible en: $<$ http:// ea.eol.org.ar/01/es/template.asp?simultaneas/iom/textos/gnapolitano.html>.

Shnurmann, A. (2002). Observación de una fobia. Bibliográfica. Biblioteca del Camp freudiá, Barcelona, 3: 31-48 (Trabalho original de 1949).

\section{Resumos}

(Fatores desencadeadores, estrutura e função do sintoma fóbico na infância: análise clínica de dois casos paradigmáticos)

O objetivo do artigo é analisar dois casos de fobias em crianças, de acordo com um método clínico: Juanito (Freud, 1909) e Sandy (Shnurmann, 1949). Os fatores desencadeadores são diferentes. Conceitualmente, no primeiro caso, é acentuada a falta paterna e, no segundo, a falta materna. A estrutura do sintoma também difere: é metafórica no primeiro caso, e, no segundo, metonímica. Em ambos os casos a função do sintoma concerne à suplência daquilo que falta.

Palavras-chave: Crianças, psicopatologia, Lacan, fobia 
(Triggering factor, structure and function of the phobic symptom in childhood: clinical analysis of two paradigmatic cases)

The aim of the article is to analyze two cases of phobias in children: Juanito (Freud, 1909) and Sandy (Shnurmann, 1949) according to the clinical method. Triggering factor differ. Conceptually in the first case it is the accentuated lack of the paternal function and, in the second case, maternal deprivation. The symptom structure also differs: it is metaphorical, in the first case, and metonymic in the second. In both cases, it concerns the substitution of the lacking function.

Key words: Children, psychopathology, Lacan, phobia

(Les facteurs de déclenchement, structure et fonction du symptôme phobique dans l'enfance: analyse clinique de deux cas paradigmatiques)

Le but de l'article est d'analyser deux cas de phobies chez les enfants: Juanito (Freud, 1909) et Sandy (Shnurmann, 1949) selon une méthode clinique. Les facteurs de déclenchement sont diffèrent dans les deux exemples. Conceptuellement dans le premier cas est accentué le manque paternel et dans le second cas le manque maternelle. La structure du symptôme diffère aussi: est métaphorique dans le premier cas et métonymique dans le second. La fonction du symptôme dans les deux cas concerne la suppléance de la fonction en défaut.

417 Mots clés: Enfants, psychopathologie, Lacan, phobie

(Trigger, Struktur und Funktion der phobische Symptome in der Kindheit: klinische Analyse von zwei paradigmatische Fälle)

Ziel des Artikels ist es, zwei Fälle von Phobien bei Kindern zu analysieren: Juanito (Freud, 1909) und Sandy (Shnurmann, 1949) nach einer klinischen Verfahren. Trigger abweichen. Konzeptionell im ersten Fall ist es Mangel väterlichen und im zweiten Fall die mütterliche Deprivation akzentuiert. Symptom-Struktur unterscheidet sich auch: ist metaphorisch im ersten Fall und dem zweiten metonymische. Funktion in beiden Fällen bezieht sich die Substitution der fehlenden Funktion.

Schlüsselwörter: Kinder, Psychopathologie, Lacan, phobia

\section{（儿童恐惧症的触发，结构和功能：两个典型病例的临床分析）}

本文的目的是临床分析法来研究兩个典型的儿童恐惧症病例, 一个患 者名叫璜尼托 (医生是弗洛伊德, 1909), 另一个名叫桑迪 (医生是史努曼 Shnurmann, 1949)。触发病情发作的因素各不相同。从概念上讲, 在第一种病 例里, 触发病情的是父爱的缺失, 在第二种病例里, 触发病情的是母爱的被剥 夺。症狀结构也有所不同: 在第一種病例中, 病人用比喻的方式对缺失的父爱进 行替代, 在第二種病例中病人用象征的方式对被剥夺的母爱进行替代。在這兩病 例里，患者都对缺失的父爱或者母爱做了替代。

关键词: 兒童, 心理病理学, 拉孔 (Lacan), 恐惧症 


\begin{abstract}
ARTIGOS
Citação/Citation: De Battista, J.; Varela, J.; Dinamarca, M. (2016, setembro). Factores desencadenantes, estructura y función del síntoma fóbico en la infancia: análisis de dos casos clínicos paradigmáticos. Revista Latinoamericana de Psicopatologia Fundamental, 19(3), 403-419.
\end{abstract}

Editores do artigo/Editors: Prof. Dr. Manoel Tosta Berlinck e Profa. Dra. Sonia Leite

Recebido/Received: 5.8.2015/ 8.5.2015 Aceito/Accepted: 19.10.2015 / 10.19.2015

Copyright: (C) 2009 Associação Universitária de Pesquisa em Psicopatologia Fundamental/ University Association for Research in Fundamental Psychopathology. Este é um artigo de livre acesso, que permite uso irrestrito, distribuição e reprodução em qualquer meio, desde que o autor e a fonte sejam citados / This is an open-access article, which permits unrestricted use, distribution, and reproduction in any medium, provided the original authors and sources are credited.

Financiamento/Funding: Esta pesquisa é financiada pela UNLP, o CONICET e Ministerio de Salud de la Provincia de Buenos Aires / This research is funded by UNLP, o CONICET e Ministerio de Salud de la Provincia de Buenos Aires.

Conflito de interesses/Conflict of interest: Os autores declaram que não há conflito de interesses / The authors have no conflict of interest to declare.

\title{
Julieta De Battista
}

Doctora en Psicopatología de la Université de Toulouse; Especialista en clínica psicoanalítica con adultos de la Universidad Nacional de La Plata; Licenciada en Psicología de la Universidad Nacional de La Plata; Profesora Adjunta a cargo de Psicopatología I UNLP.

Universidad Nacional de La Plata-CONICET; Investigadora Adjunta Asociada CIC - UNLP

Calle JA Cabrera, 5778

1414 Buenos Aires, Argentina

julietadebattista@gmail.com 


\section{Jesica Varela}

Licenciada en Psicología de la UNLP; Becaria de doctorado de la UNLP; Docente e investigadora de la cátedra de Psicopatología I. Universidad Nacional de La Plata.

Avenida Belgrano, 2701, 60 «D»

1096 Buenos Aires, Argentina

\section{Mariana Dinamarca}

Licenciada en Psicología; Becaria residente del Ministerio de Salud de la Provincia de Buenos Aires; Docente e investigadora de la cátedra de Psicopatología I. Universidad Nacional de La Plata.

Calle 55, n. 496, piso 4

1900 La Plata, Argentina

This is an open-access article, which permits unrestricted use, distribution, and reproduction in any medium for non-commercial purposes provided the original authors and sources are credited. 